\title{
Retraction: Analysis of Impact of Highly-Available Archetypes on Robotics
}

\author{
Haibo Zhu \\ Harbin University of Commerce, Harbin, China \\ haibo26@yahoo.cn
}

Several conference proceedings have been infiltrated by fake submissions generated by the SCIgen computer program. Due to the fictional content the chapter "Analysis of Impact of Highly-Available Archetypes on Robotics" by "Haibo Zhu" has been retracted by the publisher. Measures are being taken to avoid similar breaches in the future.

G. Shen and X. Huang (Eds.): ECWAC 2011, Part II, CCIS 144, pp. 109-114, 2011.

(C) Springer-Verlag Berlin Heidelberg 2011 